\title{
USING THE SEMANTIC WEB IN MOBILE AND UBIQUITOUS COMPUTING
}

\author{
Ora Lassila \\ Nokia Research Center \\ Burlington, MA, USA \\ ora.lassila@nokia.com
}

\begin{abstract}
This paper views the Semantic Web as a means to improve the interoperability between systems, applications, and information sources. Emerging personal computing paradigms such as ubiquitous and mobile computing will benefit from better interoperability, as this is an enabler for a higher degree of automation of many tasks that would otherwise require the end-users' attention. Specific application areas of Semantic Web technologies with direct ramifications to these new paradigms include Web Services, context-awareness and policy modeling.
\end{abstract}

Keywords: Semantic Web, Ubiquitous Computing, Mobile Computing, ContextAwareness, Interoperability

\section{Introduction}

The Semantic Web [Berners-Lee et al., 2001] - motivated by the rapidly growing volume of useful electronically accessible information that is only meaningful with human interpretation - is an effort to build more "machine-friendly" content (and services) for the World Wide Web. Information with accessible formal semantics can be processed by automated systems (such as autonomous agents) without human intervention or the need to apply human interpretation (which we should consider a scarce, critical resource). Deployment of the Semantic Web could ease the current human workload if it leads to easier automation of (Webbased) tasks and thus allows computers to do more on behalf of humans.

Much of the promise of the Semantic Web is predicated on the emergence of ontologies, specifications of conceptualization [Gruber, 1993] that - in essence - establish "meaning" by defining the relationships between terms of discourse and enabling reasoning as a key process through which implicit information can be uncovered. 
The emergence of standards for the Semantic Web has made it possible to start deploying the associated technologies outside research laboratory settings. Most importantly, RDF [Lassila, 1998, Lassila and Swick, 1999, Brickley and Guha, 2003] as a formalism for expressing simple taxonomical ontologies and OWL [McGuinness and van Harmelen, 2004] for more "expressive" ontologies now form the cornerstone of future Semantic Web development. These are followed by further developments for languages that allow the expression of queries [Prud'hommeaux and Seaborne, 2005] and rules [Horrocks et al., 2004].

This paper discusses the possible application of Semantic Web technologies to two new paradigms of personal computing, namely ubiquitous and mobile computing. This application is motivated by the need for better automation of user's tasks (as a means of making the user's life easier); we will adopt the view that automation is best enabled by improving the interoperability between systems, applications, and information.

\section{Enabling Interoperability}

To fully realize the vision of the Semantic Web, we must not only address representational issues but also tackle behavioral ones. Serendipitous interoperability [Lassila, 2002] - that is, the unarchitected, unanticipated encounters of agents on the Web - is an important component of this realization. Semantic Web techniques - applying knowledge representation techniques in a distributed environment - have proven useful in providing richer descriptions for Web resources. Semantic Web Services, a new research paradigm, is generally defined as the augmentation of Web Service descriptions through semantic annotations, to facilitate the higher automation of service discovery, composition, invocation and monitoring in an open, unregulated and often chaotic environment [Payne and Lassila, 2004].

Just as the success of the deployment of the Semantic Web will largely depend on whether useful ontologies will emerge, so will the Semantic Web Services benefit from mechanisms that allow shared agreements about vocabularies for knowledge representation. Sharing vocabularies allows automated interoperability; given a base ontology shared by agents, each agent can extend this ontology while achieving partial understanding of the others; this is analogous to object-oriented programming systems, where a base class defines "common" functionality.

Several activities around Semantic Web Services have emerged, the best known being the OWL-S ontology work originated in DARPA's DAML research program [Ankolekar et al., 2002, Martin et al., 2004]. 
Semantic Web Services represent an important step toward the fullblown vision of the Semantic Web, in terms of utilizing, managing and creating semantic markup.

The relationship between the Semantic Web and the current Web Service architecture depends on one's viewpoint. In the near term, the deployment of Web Services is critical; here, Semantic Web techniques can enhance the current service architecture. In the longer term, assuming the adoption of the Semantic Web vision, the deployment of Semantic Web techniques will be critical; then, Web Services will offer a ubiquitous infrastructure on which to build the next generation of inter-organizational multi-agent systems.

It is important to note that the Semantic Web represents a potential for qualitatively stronger interoperability than the traditional standardsbased approach. With the latter, one essentially has to anticipate all future scenarios, whereas in the Semantic Web approach it is possible for agents to "learn" new vocabularies and - via reasoning - make meaningful use of them. Furthermore, in addition to current notions of device and application interoperability, the Semantic Web represents interoperability at the level of the information itself.

\section{Semantic Web Meets Ubiquitous Computing}

Ubiquitous computing is an emerging paradigm for personal computing and communications [Weiser, 1991]. Although much of ubiquitous computing research has focused on user interface aspects [Abowd and Mynatt, 2000], we can argue that a characteristic of the paradigm and thereby distinctly different from the current personal computing paradigm - is the proliferation of devices that need to be connected. Today's user connects his PC to a handful of other devices (printers, network gateways, etc.) and these connections are fairly static, but we anticipate ubiquitous computing scenarios to involve dozens, if not hundreds of devices (sensors, external input and output devices, remotely controlled appliances, etc.). Furthermore, with the advent of mobility and associated proximity networking, the set of connected devices will constantly change as the usage context changes and as devices come into and leave the range of the user's ubiquitous computing device(s). Because of the dynamic nature of the new paradigm, technologies that improve interoperability will be crucial.

Given the need to dynamically connect to a large ever-changing set of devices and services, devices in a ubiquitous computing environment should be capable of sophisticated discovery and device coalition formation: the goal should be to accomplish discovery and configuration of 
new devices without "a human in the loop." In other words, the ultimate objective is the discovery and utilization of services offered by other automated systems without human guidance or intervention, thus enabling the automatic formation of device coalitions through this mechanism. Semantic Web Services, because of the benefits enabled by the application of ontological techniques (as described earlier), appears to be an appropriate paradigm to be applied in representing the functionality of ubiquitous computing devices. Virtual and physical functions can be abstracted as Web Services, providing a uniform view of all different kinds of functionality [Lassila and Adler, 2003, Masuoka et al., 2003]. Again, realization of this is contingent on the continuing emergence of suitable ontologies for modeling ubiquitous computing environments [Chen et al., 2004].

Avoiding a priori commitments about how devices are to interact with one another will improve interoperability and thus will make dynamic, unchoreographed ubiquitous computing scenarios more realistic. With reference to the aforementioned serendipitous interoperability, the true fulfillment of the vision for ubiquitous computing has a promise of serendipity in it that cannot be realized without discovery mechanisms that are qualitatively stronger than the current practice.

\section{Towards Mobile Information Access}

The advent of smartphones - mobile phones capable of functions typically associated with personal digital assistants (PDAs) or even personal computers - has made mobile information access an everyday reality. Although smartphones still suffer from various technical limitations compared to, say, laptop computers (e.g., smaller screen, slower network connectivity, often awkward keyboard input), progress is being made to make Web browsing a typical task on these devices [Nokia, 2005].

Eventually, the physical limitations inherent in mobile information access can be overcome, but we believe that the real limitations have more to do with the usage situations of mobile devices. Information access often (if not predominantly) takes place in situations where the user is "attention-constrained"; in other words, the user is primarily paying attention to something else (say, driving a car) and cannot expend full attention to the process of finding and retrieving information. Given that her attention is focused elsewhere, the mobile user may merely "have questions" and will need very specific (and thus potentially terse) answers.

A number of techniques can be applied to help focus the search and acquisition of information. For example, context awareness - the iden- 
tification of usage situations and user's tasks, and tailoring the system behavior based on these [Dey et al., 2001] - can be used to narrow the scope of user's requests. Semantic Web technologies (knowledge representation, reasoning, and the interchange of representations) are well suited to representing and processing context information [Lassila and Khushraj, 2005]. Determining context, however, typically benefits from access to as many sources of information as possible (related to the user, her task, the environment, etc.), and without a proper solution for security, privacy and trust, efforts to implement context-awareness may be hampered. Fortunately Semantic Web techniques are also well suited to describing, reasoning about, and exchanging policies which can be used to represent these [Kagal et al., 2003, Kagal, 2004]; this naturally applies to ubiquitous computing environments as well.

Generally, having access to information in "raw" form (i.e., without any forethought as to how the information is to be presented or formatted), combined with the representation and reasoning capabilities enabled by Semantic Web technologies, will be helpful, because then what information gets presented and how it gets formatted can be a context-based decision. We can think of context very broadly, covering just about everything that is known about the user, her task, the current environment, and the device she is using to access information. In this regard, it may be possible to go well beyond contemporary content repurposing approaches (such as [Nokia, 2003]). For example, it is possible to demonstrate that Semantic Web techniques can be used not only to automatically generate user interfaces from OWL-S descriptions, but that these user interfaces can be contextually optimized for small-screen devices [Khushraj and Lassila, 2005].

\section{Conclusions}

Semantic Web technologies offer several benefits to new computing paradigms such as mobile and ubiquitous computing. Not only do Semantic Web technologies lend themselves well to representation, reasoning and exchange of many different kinds of information (such as functionality, contexts, policies, user models, etc.), but generally these technologies are a qualitatively stronger approach to interoperability than contemporary standards-based approaches. With sophisticated ontological representations we can realize effortless access to heterogeneous information sources, independent of the device being used or the user's context; furthermore, we can finally untap the serendipitous potential that exists in unchoreographed encounters of automated and autonomous systems in cyberspace. 


\section{References}

[Abowd and Mynatt, 2000] Abowd, G. D. and Mynatt, E. D. (2000). Charting past, present, and future research in ubiquitous computing. ACM Transactions on Computer-Human Interaction, 7(1):29-58.

[Ankolekar et al., 2002] Ankolekar, A., Burstein, M., Hobbs, J. R., Lassila, O., McDermott, D., Martin, D., McIllraith, S. A., Narayanan, S., Paolucci, M., Payne, T., and Sycara, K. (2002). DAML-S: Web Service Description for the Semantic Web. In Horrocks, I. and Hendler, J., editors, The Semantic Web - ISWC 2002, Lecture Notes in Computer Science 2342, pages 348-363. Springer Verlag.

[Berners-Lee et al., 2001] Berners-Lee, T., Hendler, J., and Lassila, O. (2001). The Semantic Web. Scientific American, 284(5):34-43.

[Brickley and Guha, 2003] Brickley, D. and Guha, R. (2003). RDF Vocabulary Description Language 1.0: RDF Schema. W3C Working Draft, World Wide Web Consortium.

[Chen et al., 2004] Chen, H., Perich, F., Finin, T., and Joshi, A. (2004). SOUPA: Standard Ontology for Ubiquitous and Pervasive Applications. In International Conference on Mobile and Ubiquitous Systems: Networking and Services (MobiQuitous), Boston, MA.

[Dey et al., 2001] Dey, A., Abowd, G., and Salber, D. (2001). A conceptual framework and a toolkit for supporting the rapid prototyping of context-aware applications. Human-Computer Interaction, 16:97-166.

[Gruber, 1993] Gruber, T. R. (1993). A Translation Approach to Portable Ontology Specifications. Knowledge Acquisition, 5(2):199-220.

[Horrocks et al., 2004] Horrocks, I., Patel-Schneider, P. F., Boley, H., Tabet, S., Grosof, B., and Dean, M. (2004). SWRL: A Semantic Web Rule Language Combining OWL and RuleML. W3C Member Submission, World Wide Web Consortium.

[Kagal, 2004] Kagal, L. (2004). A Policy-Based Approach to Governing Autonomous Behavior in Distributed Environments. PhD thesis, University of Maryland Baltimore County, Baltimore MD.

[Kagal et al., 2003] Kagal, L., Parker, J., Chen, H., Joshi, A., and Finin, T. (2003). Security, Privacy and Trust in Mobile Computing Environments, chapter Security and Privacy Aspects. CRC Press.

[Khushraj and Lassila, 2005] Khushraj, D. and Lassila, O. (2005). Ontological Approach to Generating Personalized User Interfaces for Web Services. To appear.

[Lassila, 1998] Lassila, O. (1998). Web Metadata: A Matter of Semantics. IEEE Internet Computing, 2(4):30-37.

[Lassila, 2002] Lassila, O. (2002). Serendipitous Interoperability. In Eero Hyvönen, editor, The Semantic Web Kick-off in Finland - Vision, Technologies, Research, and Applications, HIIT Publications 2002-001. University of Helsinki.

[Lassila and Adler, 2003] Lassila, O. and Adler, M. (2003). Semantic Gadgets: Ubiquitous Computing Meets the Semantic Web. In Fensel, D., Hendler, J., Wahlster, W., and Lieberman, H., editors, Spinning the Semantic Web, pages 363-376. MIT 
[Lassila and Khushraj, 2005] Lassila, O. and Khushraj, D. (2005). Contextualizing Applications via Semantic Middleware. Accepted to The Second Annual International Conference on Mobile and Ubiquitous Systems: Networking and Services (MobiQuitous).

[Lassila and Swick, 1999] Lassila, O. and Swick, R. R. (1999). Resource Description Framework (RDF) Model and Syntax Specification. W3C Recommendation, World Wide Web Consortium.

[Martin et al., 2004] Martin, D., Burstein, M., Hobbs, J., Lassila, O., McDermott, D., Mcllraith, S., Narayanan, S., Paolucci, M., Parsia, B., Payne, T., Sirin, E., Srinivasan, N., and Sycara, K. (2004). OWL-S: Semantic Markup for Web Services. W3C Member Submission, World Wide Web Consortium.

[Masuoka et al., 2003] Masuoka, R., Parsia, B., and Labrou, Y. (2003). Task Computing - the Semantic Web meets Pervasive Computing. In Proceedings of the 2nd International Semantic Web Conference 2003 (ISWC 2003).

[McGuinness and van Harmelen, 2004] McGuinness, D. L. and van Harmelen, F. (February 2004). OWL Web Ontology Language Overview. W3C Recommendation, World Wide Web Consortium.

[Nokia, 2003] Nokia (2003). Nokia acquires Eizel to enhance mobile enterprise portfolio. Nokia press release, 2003-04-22.

[Nokia, 2005] Nokia (2005). Nokia develops a new browser for Series 60 by using open source software. Nokia press release, 2005-06-13.

[Payne and Lassila, 2004] Payne, T. and Lassila, O. (2004). Semantic Web Services (guest editors' introduction). IEEE Intelligent Systems, 19(4):14-15.

[Prud'hommeaux and Seaborne, 2005] Prud'hommeaux, E. and Seaborne, A. (2005). SPARQL Query Language for RDF. W3C Working Draft, World Wide Web Consortium.

[Weiser, 1991] Weiser, M. (1991). The computer for the twenty-first century. Scientific American, 265(3):94-104.

\section{Acknowledgements}

The author's own research cited in this paper was supported in part by the Nokia Technology Platforms and the Nokia Research Center. 